\title{
A NEW CRITERION FOR COMPLETELY MONOTONIC FUNCTIONS
}

\author{
BY \\ HARRY POLLARD
} $C^{\infty}$ and

A function $f(x)$ is completely monotonic (c.m.) in $0<x<\infty$ if it belongs to

$$
(-1)^{k} f^{(k)}(x) \geqq 0
$$$$
(k \geqq 0, x>0) \text {. }
$$

If $f(x)$ can be extended to be continuous at $x=0$ it is said to be c.m. in $0 \leqq x<\infty$.

Various conditions are known under which a function is c.m. [4]( $\left.{ }^{1}\right)$. Bernstein proved that if

$$
(-1)^{k} \Delta_{h}^{k} f(x)=\sum_{0}^{k} C_{k, n}(-1)^{n} f(x+n h) \geqq 0 \quad(k \geqq 0, x>0, h>0)
$$

then $f(x)$ is c.m. in $0<x<\infty$. It is known, though apparently not stated explicitly in the literature, that if we assume the continuity of $f(x)$, then we need require (2) only for some infinite sequence of integers $k$. (This may be obtained, for example, by use of the results of [1].) A fundamental theorem of Bernstein and Widder states that a function is c.m: in $0<x<\infty$ if and only if it admits the representation

$$
f(x)=\int_{0}^{\infty} e^{-x t} d F(t), \quad x>0, F(t) \text { increasing. }
$$

A new difference criterion which includes the above is suggested by the following considerations. If $h_{k}=o\left(1 / k^{2}\right)$ then (3) is inverted by [3, Theorem 4.2],

where

$$
F(t)-F(0)=f(\infty)+\lim _{k \rightarrow \infty} d_{k} \int_{k / t}^{\infty} x^{k-1} \Delta_{h_{k}}^{k} f(x) d x
$$

$$
d_{k}=\left(-h_{k}\right)^{-k} /(k-1) !
$$

This suggests the following theorem, which is the principal result of this paper.

THEOREM. Let $f(x)$ be continuous for $x \geqq 0$ and have a limit at infinity. Suppose it satisfies the inequalities

Presented to the Society, September 13, 1943; received by the editors August 30,1943, and, in revised form, November 1, 1943.

(1) Numbers in brackets refer to the references listed at the end of the paper. 


$$
(-1)^{k} \Delta_{h_{k}}^{k} f(x) \geqq 0
$$

for an infinite sequence of integers $k$, where

$$
h_{k}>0, \quad h_{k}=o\left(1 / k^{2}\right)
$$

Then $f(x)$ is c.m. in $0 \leqq x<\infty$.

This says essentially that in the difference criteria it is sufficient that the inequalities hold for one (suitable) value of $h$ for each $k$, rather than all $h>0$.

Before proceeding to the proof we make some observations about the theorem.

(i) The conditions are trivially necessary.

(ii) The continuity of $f(x)$ is not a redundant condition. For let $\phi(x)$ be a discontinuous solution of the functional equation $\phi(x+y)=\phi(x)+\phi(y)[2, p$. 96]. Both $\pm \phi(x)$ are convex and hence unbounded in every interval [2, pp. 91-92]. Then (5) is satisfied for arbitrarily small $\left\{h_{k}\right\}$ by the function $f(x)=e^{\phi(x)}$.

1. Lemmas. The following identity is known [4, p. 303].

Lemma 1. For $k>0, x \geqq 0, u>0$,

$$
\frac{\partial^{k}}{\partial u^{k}}\left[e^{-k x / u} u^{k-1}\right]=\frac{1}{u}\left(\frac{k x}{u}\right)^{k} e^{-k x / u} .
$$

For fixed $x$, these functions are increasing in $0 \leqq u \leqq x / 2$.

LEMMA 2. If $g(x)$ and $r(x)$ are any functions of $x$ then

$$
\Delta_{h}^{k}[g(x) r(x)]=\sum_{0}^{k} C_{k, n} \Delta_{h}^{n} g(x+\overline{k-n} h) \Delta_{h}^{k-n} r(x) .
$$

This is the analogue of Leibniz' rule for the differentiation of a product and can be established by induction.

Lemma 3 (Generalized Rolle's theorem). If $f(x) \in C^{k}$ then

$$
\Delta_{h}^{k} f(x)=h^{k} f(k)(X)
$$

where $X$ lies between $x$ and $x+k h$.

LEMMA 4. Suppose $k \geqq 1, x>0, h>0$ are fixed, and $f(u)$ is continuous for $u \geqq k h, f(\infty)=0$. Then

$$
\lim _{\leftrightarrow \rightarrow 0} \int_{k h}^{\infty} \Delta_{-h}^{k}\left[e^{-\epsilon u} e^{-k x / u} u^{k-1}\right] f(u) d u=\int_{k h}^{\infty} \Delta_{-h}^{k}\left[e^{-k x / u} u^{k-1}\right] f(u) d u
$$

where we difference with respect to $u$. 
Proof. The existence of the integral on the left is guaranteed by the presence of the factor $e^{-\imath u}$. The integral on the right converges since, by virtue of Lemmas 3 and 1, its integrand is dominated for large $u$ by $(u-k h)^{-k-1}|f(u)|$.

If we subtract the right-hand side the problem is then to show that

$$
H(\epsilon)=\int_{k h}^{\infty} \Delta_{h}^{k}[g(u) r(u)] f(u) d u=o(1) \quad(\epsilon \rightarrow 0+),
$$

where

$$
g(u)=e^{-k x / u} u^{k-1}, r(u)=1-e^{-\varepsilon u} .
$$

Using Lemma 2 with $h$ replaced by $-h$ and separating out the term for which $n=k$ we obtain

$$
\begin{aligned}
H(\epsilon)= & \sum_{n=0}^{k-1} C_{k, n} \int_{k h}^{\infty} \Delta_{-h}^{n} g(u-\overline{k-n} h) \cdot \Delta_{-h}^{k-n} r(u) \cdot f(u) d u \\
& +\int_{k h}^{\infty} \Delta_{-h}^{k} g(u) \cdot r(u) \cdot f(u) d u \\
= & K(\epsilon)+L(\epsilon) .
\end{aligned}
$$

By Lemmas 3 and 1 the integral $\int_{k h}^{\infty} \Delta_{-h}^{k} g(u) \cdot f(u) d u$ exists. Hence

$$
L(\epsilon)=\int_{k h}^{\infty}\left(1-e^{-\epsilon u}\right) \Delta_{-h}^{k} g(u) f(u) d u=o(1) \quad(\epsilon \rightarrow 0+) .
$$

We turn now to the expression $K(\epsilon)$. First by Lemma 3

$$
\Delta_{-h}^{k-n} r(u)=(-h)_{r}^{k-n}{ }^{(k-n)}(U)=(h \epsilon)^{k-n} e^{-\epsilon U}, \quad u-(k-n) h \leqq U \leqq u,
$$

so that for $0<\epsilon<1$

$$
\left|\Delta_{-h}^{k-n} r(u)\right| \leqq K_{1} \epsilon^{k-n} e^{-\epsilon u},
$$

where $K_{1}$ does not depend on $u$ or $\epsilon$. Also

$$
\Delta_{-h g}^{n} g(u-\bar{k}-n h)=(-h)^{n} g^{(n)}(U), \quad u-k h \leqq U \leqq u-(k-n) h .
$$

But

If $x>0$

$$
g^{(n)}(u)=\sum_{j=0}^{n} C_{n, j} \frac{\partial^{j}}{\partial u^{j}}\left[e^{-k x / u}\right]\left[u^{k-1}\right]^{(n-j)}
$$

so that

$$
\frac{\partial^{j}}{\partial u^{j}}\left[e^{-k x / u}\right]=O\left(u^{-j}\right)
$$

$$
g^{(n)}(u)=\sum O\left(u^{-i} u^{k-1-n+j}\right)=O\left(u^{k-n-1}\right), \quad 0 \leqq n \leqq k-1 .
$$


If $x=0$ this result is obvious. Then by (9)

$$
\left|\Delta_{-h}^{n} g(u-\overline{k-n} h)\right| \leqq K_{2} u^{k-n-1} \quad(0 \leqq n \leqq k-1),
$$

where $K_{2}$ does not depend on $u$. Then by (7), (8), (10)

$$
|K(\epsilon)| \leqq K_{1} K_{2} \sum_{n=0}^{k-1} C_{k, n} \int_{k h}^{\infty} \epsilon^{k-n} e^{-\epsilon u} u^{k-n-1}|f(u)| d u \quad(0<\epsilon<1) .
$$

Since $f(\infty)=0$ a simple Abelian argument proves that each term of the sum approaches zero with $\epsilon$. Hence $K(0+)=0$, so $H(0+)=0$; this establishes (6).

Lemma 5. Let $k \geqq 1, h>0, x \geqq 0$ be fixed, $f(u)$ continuous, $0 \leqq u<\infty$, $f(\infty)=0$. If $\Delta_{h}^{k} f(u)$ does not change sign in $0 \leqq u<\infty$, then

$$
\begin{aligned}
\int_{0}^{\infty} e^{-k x / u} u^{k-1} \Delta_{h}^{k} f(u) d u & \\
= & \sum_{n=0}^{k} C_{k, n}(-1)^{k-n} \int_{n h}^{k h} e^{-k x /(u-n h)}(u-n h)^{k-1} f(u) d u \\
& +\int_{k h}^{\infty} \Delta_{-h}^{k}\left[e^{-k x / u} u^{k-1}\right] \cdot f(u) d u,
\end{aligned}
$$

where we difference with respect to $u$.

Clearly the integral

$$
I(\epsilon)=\int_{0}^{\infty} e^{-\epsilon u} e^{-k x / u} u^{k-1} \Delta_{h}^{k} f(u) d u
$$

exists for all $\epsilon>0$. We have

$$
\begin{aligned}
& I(\epsilon)=\sum_{0}^{k} C_{k, n}(-1)^{k-n} \int_{0}^{\infty} e^{-\epsilon u} e^{-k x / u} u^{k-1} f(u+n h) d u \\
&=\sum_{0}^{k} C_{k, n}(-1)^{k-n}\left(\int_{n h}^{k h}+\int_{k h}^{\infty}\right) \exp [-\epsilon(u-n h)-k x /(u-n h)] \\
& \cdot(u-n h)^{k-1} f(u) d u,
\end{aligned}
$$

obtained by a change of variable. Then

$$
I(\epsilon)=A(\epsilon)+B(\epsilon),
$$

where

$$
\begin{aligned}
& A(\epsilon)=\sum_{0}^{k} C_{k, n}(-1)^{k-n} \int_{n h}^{k h} \exp [-\epsilon(u-n h)-k x /(u-n h)] \\
& B(\epsilon)=\int_{k h}^{\infty} \Delta_{-h}^{k}\left[e^{-\epsilon u} e^{-k x / u} u^{k-1}\right] f(u) d u .
\end{aligned}
$$


By dominated convergence

$$
A(0+)=\sum_{0}^{k} C_{k, n}(-1)^{k-n} \int_{n h}^{k h} e^{-k x /(u-n h)}(u-n h)^{k-1} f(u) d u,
$$

and by Lemma 4

$$
B(0+)=\int_{k h}^{\infty} \Delta_{-h}^{k}\left[e^{-k x / u} u^{k-1}\right] \cdot f(u) d u .
$$

From (12), (13), (14) it follows that $I(0+)$ exists and equals the expression on the right-hand side of (11). Since $\Delta_{h}^{k} f(u)$ does not change sign in $0 \leqq u<\infty$, a Tauberian theorem enables us to conclude that $I(0+)$ is also equal to the left-hand side of (11) $[4$, p. 192].

For the remainder of the paper it is assumed that $k$ belongs to some sequence $S$ of non-negative integers; $k \rightarrow \infty$ means that $k$ becomes infinite through the elements of $S$.

Lemma 6. Let $f(x)$ satisfy all the hypotheses of our theorem and suppose also that $f(\infty)=0$. Then for any fixed $x>0$ the quantities

$$
I_{k}=d_{k} \int_{0}^{\infty} e^{-k x / u} u^{k-1} \Delta_{h_{k}}^{k} f(u) d u
$$

approach $f(x)$ as $k \rightarrow \infty$. The $d_{k}$ are defined as in (4).

Proof. By Lemma 5 we have $I_{k}=A_{k}+B_{k}$ where

$$
\begin{aligned}
A_{k} & =d_{k} \sum_{0}^{k} C_{k, n}(-1)^{k-n} \int_{n h_{k}}^{k h_{k}} e^{-k x /\left(u-n h_{k}\right)}\left(u-n h_{k}\right)^{k-1} f(u) d u, \\
B_{k} & =d_{k} \int_{k h_{k}}^{\infty} \Delta_{-h_{k}}^{k}\left[e^{-k x / u} u^{k-1}\right] f(u) d u .
\end{aligned}
$$

We show first that $A_{k}$ vanishes with $1 / k$. Let $M$ be the maximum of $|f(x)|$ in $(0, \infty)$. Then

$$
\begin{aligned}
\left|A_{k}\right| & \leqq M\left|d_{k}\right| \sum_{0}^{k} C_{k, n} \int_{n h_{k}}^{k h_{k}} e^{-k x\left(u-n h_{k}\right)}\left(u-n h_{k}\right)^{k-1} d u \\
& \leqq M\left|d_{k}\right| \sum C_{k, n} \int_{0}^{(k-n) h_{k}} e^{-k x / u} u^{k-1} d u \\
& \leqq M\left|d_{k}\right| \sum C_{k, n} \int_{0}^{k h_{k}} e^{-k x / u} u^{k-1} d u \\
& \leqq M\left|d_{k}\right| e^{-x / h_{k}} \sum_{0}^{k} C_{k, n} \int_{0}^{k h_{k}} u^{k-1} d u \\
& =M e^{-x / h_{k} 2^{k} k^{k} / k !}
\end{aligned}
$$


Choose $k_{0}$ so that $k h_{k}<x / 2$ for $k>k_{0}$. Then for $k>k_{0}$

$$
\left|A_{k}\right| \leqq M e^{-2 k} 2^{k} k^{k} / k ! \text {. }
$$

By the test-ratio test this last is the general term of a convergent series, so that $A_{k}=o(1), k \rightarrow \infty$.

We must prove then that

$$
\lim _{k \rightarrow \infty} B_{k}=f(x)
$$

By Lemmas 3 and 1, and equation (4),

$$
\begin{aligned}
B_{k} & =\frac{1}{(k-1) !}\left(\int_{x / 2}^{\infty}+\int_{k h_{k}}^{x / 2}\right)\left[\frac{1}{u}\left(\frac{k x}{u}\right)^{k} e^{-k x / u}\right]_{u=v-\phi_{k}} f(v) d v \\
& =C_{k}+D_{k},
\end{aligned}
$$

where

$$
0 \leqq \phi_{k} \leqq k h_{k} .
$$

By the second part of Lemma 1

$$
\begin{aligned}
\left|D_{k}\right| & \leqq \frac{1}{(k-1) !}\left[\frac{1}{u}\left(\frac{k x}{u}\right)^{k} e^{-k x / u}\right]_{u=x / 2} \int_{0}^{x / 2}|f(v)| d v & \\
& =o(1) & (k \rightarrow \infty) .
\end{aligned}
$$

Our problem then is to show that $\lim _{k \rightarrow \infty} C_{k}=f(x)$. But it is known that [4, p. 283]

$$
J_{k}=\frac{1}{(k-1) !} \int_{x / 2}^{\infty} \frac{1}{u}\left(\frac{k x}{u}\right)^{k} e^{-k x / u} f(u) d u \rightarrow f(x), \quad k \rightarrow \infty .
$$

It therefore remains only to show that $\lim _{k \rightarrow \infty}\left(J_{k}-C_{k}\right)=0$ and the proof of the lemma will be complete.

Now

$$
J_{k}-C_{k}=\frac{1}{(k-1) !} \int_{x / 2}^{\infty} \frac{1}{u}\left(\frac{k x}{u}\right)^{k} e^{-k x / u} f(u) P_{k}(u) d u
$$

where

$$
P_{k}(u)=1-\left(\frac{u}{u-\phi_{k}}\right)^{k+1} \exp \left(-\frac{k x \phi_{k}}{u\left(u-\phi_{k}\right)}\right) \quad(u \geqq x / 2) .
$$

We have

$$
\begin{aligned}
\log \left(1-P_{k}(u)\right) & =(k+1) \int_{u-\phi_{k}}^{u} \frac{d v}{v}-\frac{k x \phi_{k}}{u\left(u-\phi_{k}\right)}, \\
\left|\log \left(1-P_{k}(u)\right)\right| & \leqq \frac{(k+1) \phi_{k}}{u-\phi_{k}}+\frac{k x \phi_{k}}{u\left(u-\phi_{k}\right)} .
\end{aligned}
$$


By (15) we have $0 \leqq \phi_{k} \leqq k h_{k}$. Since $h_{k}=o\left(1 / k^{2}\right), \phi_{k}<x / 4$ for $k>k_{0}$. It follows that for $u \geqq x / 2, k>k_{0}$,

$$
\begin{aligned}
\left|\log \left(1-P_{k}(u)\right)\right| & \leqq \frac{(k+1) k h_{k}}{x / 2-x / 4}+\frac{k x \cdot k h_{k}}{(x / 2)(x / 2-x / 4)} \\
& =o(1)
\end{aligned}
$$

uniformly for $u \geqq x / 2$.

Let $\epsilon>0$ be arbitrary. Then for $k>k_{1}$,

By (16)

$$
\left|P_{k}(u)\right|<\epsilon \quad(u \geqq x / 2) .
$$

$$
\left|J_{k}-C_{k}\right|<\frac{\epsilon}{(k-1) !} \int_{x / 2}^{\infty} \frac{1}{u}\left(\frac{k x}{u}\right)^{k} e^{-k x / u}|f(u)| d u \text {. }
$$

As $k \rightarrow \infty$ the right-hand side approaches $\epsilon|f(x)|[4$, p. 283]. Hence

$$
\limsup _{k \rightarrow \infty}\left|J_{k}-C_{k}\right| \leqq \epsilon|f(x)| \text {, }
$$

and this completes the proof.

2. Proof of the theorem. We may assume $f(\infty)=0$ (otherwise consider $f(x)-f(\infty))$. By hypothesis

$$
L_{k, t}[f]=\left(\left(-h_{k}\right)^{-k} / k !\right)\left[x^{k+1} \Delta_{h_{k}}^{k} f(x)\right]_{x=k / t} \geqq 0
$$

for an infinite sequence of integers $k$. By Lemma 6 with a change of variable we have

$$
f(x)=\lim _{x \rightarrow \infty} \int_{0}^{\infty} e^{-x t} L_{k, t}[f] d t
$$

It remains only to show that the integrals

$$
L_{k}=\int_{0}^{\infty} L_{k, t}[f] d t
$$

exist and are bounded. For then it will follow by a familiar argument that $f(x)$ has the representation (3) $[4$, p. 307]. From Lemma 5 with $x=0$ it follows that

$$
\begin{aligned}
L_{k} & =d_{k} \sum_{0}^{k} C_{k, n}(-1)^{k-n} \int_{n h_{k}}^{k h_{k}}\left(u-n h_{k}\right)^{k-1} f(u) d u \\
& =\left(-h_{k}\right)^{-k} \Delta_{k_{k}}^{k} F_{k}(0)
\end{aligned}
$$

where

$$
F_{k}(x)=\frac{1}{(k-1) !} \int_{x}^{k h_{k}}(u-x)^{k-1} f(u) d u
$$




\section{By Lemma 3}

$$
L_{k}=(-1)^{k} F_{k}^{(k)}\left(X_{k}\right), \quad 0 \leqq X_{k} \leqq k h_{k} .
$$

But $F_{k}^{(k)}(x)=(-1)^{k} f(x)$, so that $L_{k}=f\left(X_{k}\right)$ and $\lim _{k \rightarrow \infty} L_{k}=f(0)$. Then $L_{k}$ is bounded.

\section{REFERENCES}

1. R. P. Boas and D. V. Widder, Functions with positive differences, Duke Math. J. vol.7 (1940) pp. 496-503.

2. G. H. Hardy, J. E. Littlewood, and G. P6lya, Inequalities, Cambridge, 1934.

3. H. Pollard, Real inversion formulas for the Laplace integral, Duke Math. J. vol. 7 (1940) pp. 445-451.

4. D. V. Widder, The Laplace transform, Princeton, 1941.

Kenyon College,

Gambier, OHIO 